\section{NEW TOOTHPASTE ACTIVELY STRENGTHENS CHILDREN'S TEETH}

A new toothpaste for children aged three to six which actively strengthens and protects teeth while it cleans them has been launched in the UK and Europe. Developed by BioMin technologies, the toothpaste - BioMin F for Kids - is being released in two flavours and contains a 'smart effect' that means it is especially effective when children consume acidic foods and drinks.

BioMin $\mathrm{F}$ for Kids is based on the same clinically proven technology as BioMin F, helping to strengthen and protect children's teeth and preventing them from damage.

Traditional fluoride toothpastes become washed away by saliva in a relatively short time while, in contrast, BioMin's 'smart technology' controls the release of fluoride for up to 12 hours after brushing.

Professor Robert Hill worked with scientist colleagues at Queen Mary University, London to develop the toothpaste.

BioMin F for Kids contains a polymer that adheres the BioMin particles to the teeth which is slowly dissolved by saliva, continuously releasing an optimum mix of fluoride, calcium and phosphate ions to produce fluorapatite on the tooth surfaces. It is this controlled release mechanism which means that a much lower quantity of fluoride is needed to have a greater effect.
At the same time the 'smart-effect' means that in the presence of acidic food and drink in the mouth, the toothpaste starts dissolving more rapidly. This restores the mineral equilibrium and starts the remineralisation process.

Launched in two flavours, melon and strawberry, the toothpaste is available to buy directly from BioMin's distributors list on https://www.BioMin.co.uk/wherebuy. To make brushing fun and to support better oral health in children, BioMin has created 'Bino', a friendly 'binosaur' as well as developing colouring-in sheets, stickers and a useful brushing chart.

BioMin also offers two toothpastes for teenagers and adults, BioMin F and BioMin $\mathrm{C}$ (fluoride-free) which reduce sensitivity and strengthen and protect the tooth surfaces.

BioMin F for Kids has undergone all the appropriate cytotoxicity and biocompatibility studies ensuring its safety. It is not tested on animals and is suitable for vegans and has halal certification. The fluoride concentration at $530 \mathrm{ppm}$ is the same as the adult version, which is safe for children of three years (or those weighing at least $10 \mathrm{~kg}$ ) and above. The toothpaste is available in a silvery gel format which does not contain titanium dioxide.

www.BioMin.co.uk

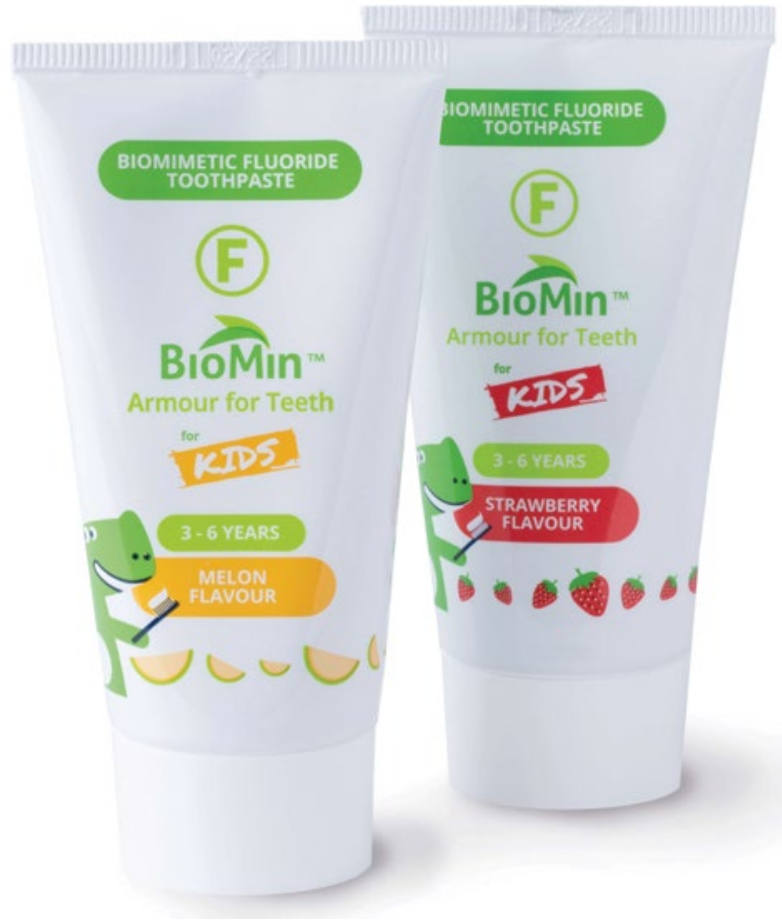

\section{FOR PATIENT CONFIDENCE IN YOUR PRACTICE}

When you welcome patients back to the practice, you want them to feel safe.

Reinforce your infection control protocols with the Steri-7 Xtra range from Initial Medical, a high-quality cleaning and disinfectant solution for surfaces and hands.

Steri-7 Xtra High Level Surface Disinfectant Cleaner is available as a concentrated solution, a ready to use spray, and a surface wipe. The Steri-7 Xtra Personal Care Range includes a hypoallergenic surgical level hand rub and wash, both of which are effective and kind to skin.

Steri-7 Xtra deactivates up to 99.999\% of a broad range of pathogens within seconds of contact and has been tested against feline Coronavirus: a surrogate for Coronavirus. Steri-7 Xtra surface sanitisers utilise specially formulated micro emulsion technology that applies an active barrier which can persist and kills for hours afterward. Initial Medical's supplier's laboratory testing showed the barrier killing microbes (including pathogens) 72 hours after application, so long as the barrier is not wiped away.

With Steri-7 Xtra, your patients can be confident about how protected they are.

You can buy the Steri-7 Xtra range online: https://www.initial.co.uk/ infection-control/surface-hygiene/.

For further information visit www. initial.co.uk/medical or tel: 0870850 4045.

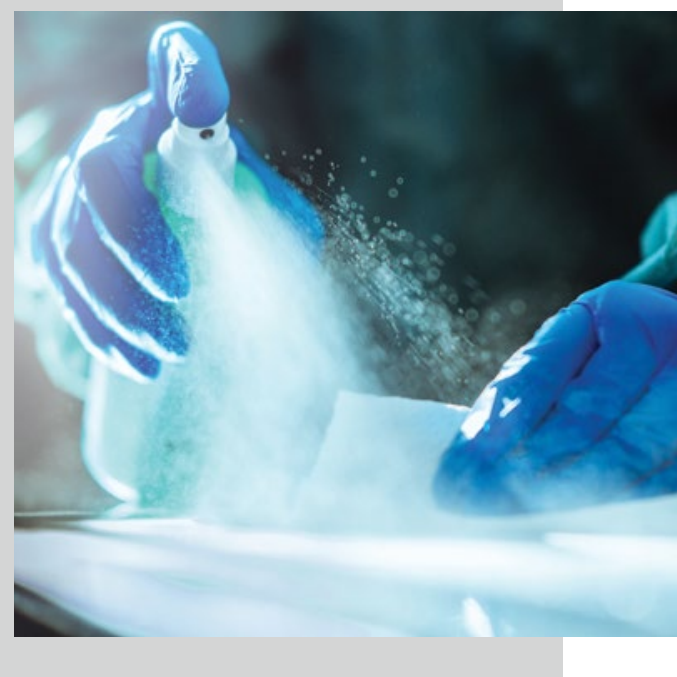

